\title{
Carbon Nanotubes Immersed in Superfluid Helium: The Impact of Quantum Confinement on Wetting and Capillary Action
}

\author{
Andreas W. Hauser* ${ }^{* \dagger}$ and María Pilar de Lara-Castells* ${ }^{*} \ddagger$ (ㅇ) \\ ${ }^{\dagger}$ Institute of Experimental Physics, Graz University of Technology, Petersgasse 16, 8010 Graz, Austria \\ ${ }^{\ddagger}$ Instituto de Física Fundamental, CSIC, Serrano 123, 28006 Madrid, Spain
}

Supporting Information

ABSTRACT: A recent experimental study [Ohba, Sci. Rep. 2016, 6 , 28992] of gas adsorption on single-walled carbon nanotubes at temperatures between 2 and $5 \mathrm{~K}$ reported a quenched propagation of helium through carbon nanotubes with diameters below $7 \AA$ despite the small kinetic diameter of helium atoms. After assessing the performance of a potential model for the $\mathrm{He}$-nanotube interaction via $\mathrm{ab}$ initio calculations with density functional theorybased symmetry adapted perturbation theory, we apply orbital-free helium density functional theory to show that the counterintuitive experimental result is a consequence of the exceptionally high zeropoint energy of helium and its tendency to form spatially separated layers of helium upon adsorption at the lowest temperatures. Helium filling factors are derived for a series of carbon nanotubes and compared to the available experimental data.

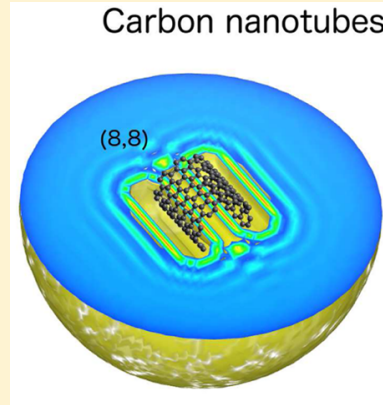

stays hollow

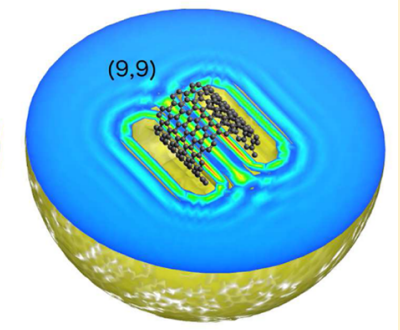

gets filled
C arbon-based nanoporous materials have attracted much attention in recent years because of their outstanding adsorption capabilities with potential applications for gas storage and separation. ${ }^{1}$ Among them, single-walled nanotubes stand out because of their one-dimensional flow channels, which can be adjusted in terms of helicity, channel length, and diameter. ${ }^{2,3}$ The process of gas adsorption and the propagation through porous materials with diameters in the nanometer regime is typically described by molecular dynamics simulations. The most obvious limitation of this approach concerns the appearance of quantum phenomena such as zero-point energy or quantum tunneling effects, which become relevant for light particles at the lowest temperatures. Very recent examples are isotope separations via carbon nanotubes, ${ }^{4}$ graphene membranes, ${ }^{5-7}$ graphdiyne, ${ }^{8,9}$ or carbon materials in general, ${ }^{10}$ which are typically treated with more advanced techniques such as ring polymer molecular dynamics ${ }^{11,12}$ or via first-principles methods.

A series of interesting questions arises if supramolecular structures are embedded in a quantum fluid at temperatures near absolute zero Kelvin. At these conditions, helium atoms are known to adsorb to a substrate in a layerlike structure due to the hard-core repulsion between individual atoms. ${ }^{13-17}$ Stronger binding surfaces such as graphite or graphene create large local pressures which even lead to solidification of the first few layers, an effect which reveals itself in pronounced density fluctuations in the normal direction to the surface. ${ }^{16,18}$ These phenomena should also affect the hydrodynamic behavior of superfluid helium when propagating through nanopores, as has been experimentally investigated recently. ${ }^{19,20}$ A series of theoretical studies was dedicated to the study of quantum films, using various approaches such as the hypernetted EulerLagrange method, ${ }^{15,21-23}$ path-integral Monte Carlo simulations, ${ }^{18,24-27}$ or helium density functional theory (DFT). ${ }^{14,16,28-30}$ The latter two approaches have also been extensively applied to the study of superfluid helium nanodroplets, which are a common experimental tool for atomic and molecular spectroscopy in a weakly perturbing environment. $^{31-34}$

In this Letter, we simulate the embedding of single-walled nanotubes of various diameters into liquid helium at a temperature of $0.38 \mathrm{~K}$. Our simulations comprise nanotube structures of up to 360 carbon atoms embedded into a helium nanodroplet composed of 2000 helium atoms. Because the interaction of atomic carbon with $\mathrm{He}$ is stronger than the interaction between two $\mathrm{He}$ atoms, the "heliophilic" carbon materials are supposed to fully submerge after embedding. However, because nanotubes are hollow objects, their sinking will be preceded by the flooding of the interior, similar to the sinking of an open metal can in water. In the classical picture, this is only hindered or delayed by the presence of gas inside, which has to escape the hollow object first. This is obviously not the case here; instead, the helium inside the nanotube will be strongly constrained in two out of three dimensions, which leads to additional energy costs due to the increased zero-point energy. As a consequence, a hindered or incomplete flooding is

Received: October 17, 2016

Accepted: November 15, 2016

Published: November 15, 2016 


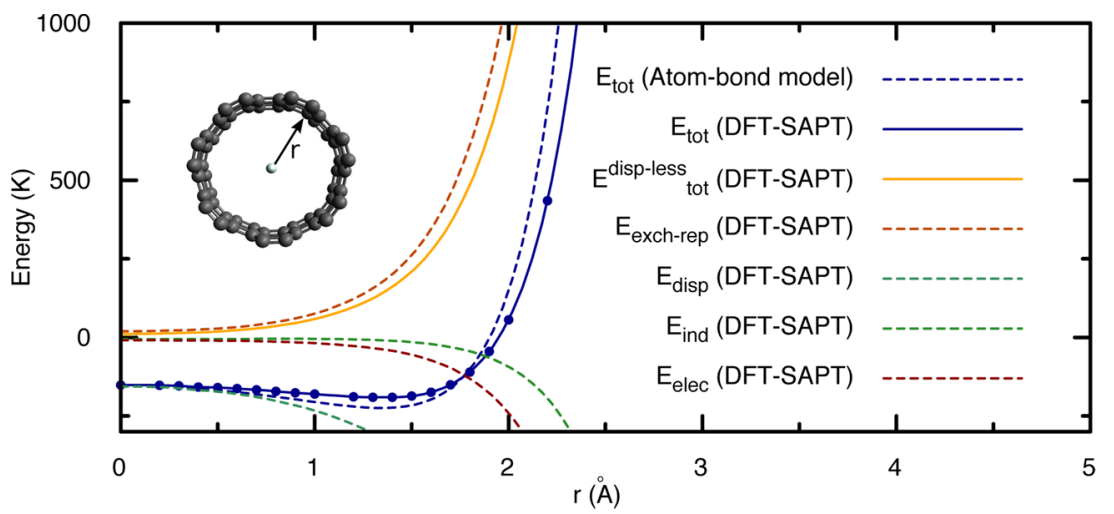

Figure 1. DFT-SAPT-based decomposition of the $\operatorname{He} / \mathrm{CNT}(7,7)$ interaction energy in dispersionless (exchange-repulsion, electrostatic, and induction) and dispersion contributions for a single He atom located at a distance $r$ from the nanotube mass center and at the central plane perpendicular to the nanotube long axis. A comparison between atom-bond model and DFT-SAPT potential energy curves is also shown.
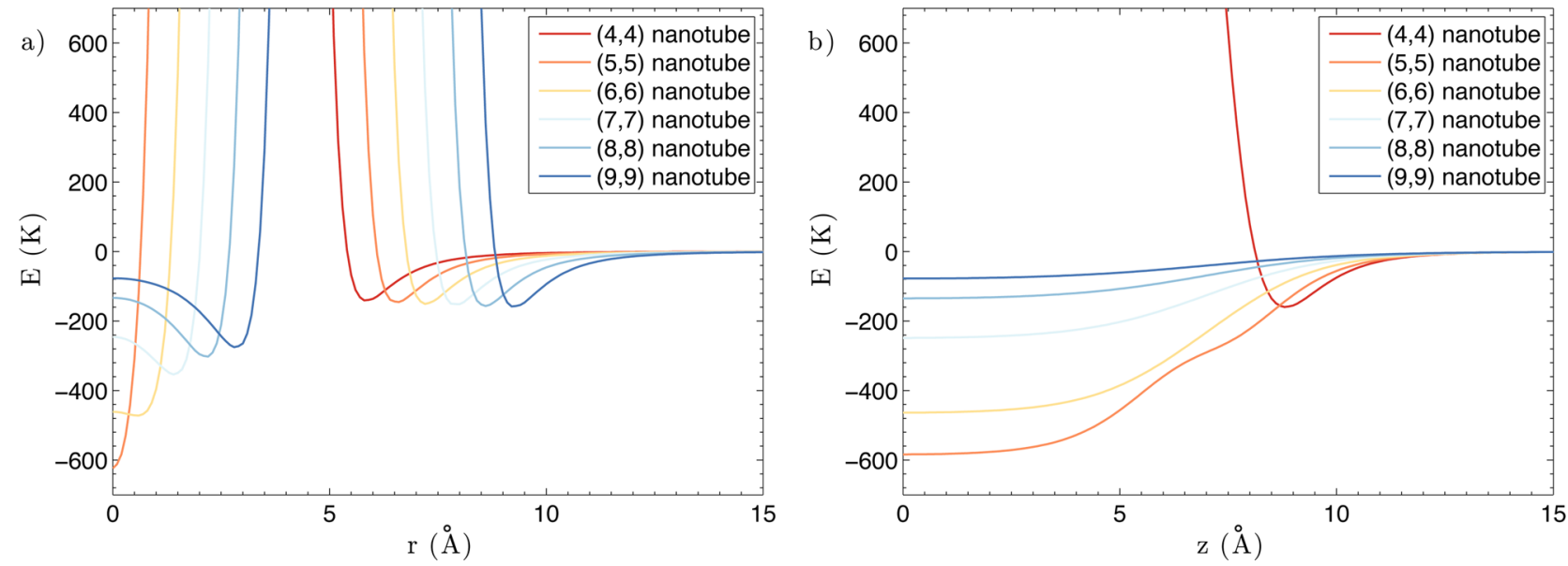

Figure 2. Radial (a) and axial scans (b) of the PES for the interaction of a single He atom with carbon nanotubes of various size. Only for the $(5,5)$ nanotube is the potential minimum on the $z$-axis. For all larger nanotubes the minimum lies near the inner surface. Note that the inside of the $(4,4)$ nanotube is not accessible to helium.

assumed for nanotubes of sufficiently small diameter. However, because it is, in contrast to the macroscopic analogy we chose, not gravity which pulls the object into the liquid but simply the interaction between helium and the nanotube, the latter will also sink into the liquid, even if the inside of the carbon nanotube (CNT) is not fully filled by helium. We will prove this thought experiment in two steps: one dedicated to the generation of suitable interaction potentials from first principles, and one to the calculation of actual $\mathrm{He}$ density distributions based on these potentials.

We start with the potential felt by a single $\mathrm{He}$ atom inside a carbon nanotube of a given radius. Our method of choice is DFT-based symmetry-adapted perturbation theory (DFTSAPT $)^{35,36}$ as implemented in Molpro, ${ }^{37}$ which we apply to short carbon nanotubes with the smaller helicity indices $(5,5)$ and $(7,7)$ because of computational limitations; see the Supporting Information for details. Figure 1 shows the weak attractive interaction experienced by a single $\mathrm{He}$ atom with the inner walls of a carbon nanotube, which results from a subtle balance between dispersionless (mostly exchange-repulsion) and dispersion energy contributions. Note that the dependence of this interplay on the nanotube radius is an important feature of the $\mathrm{He}-\mathrm{CNT}$ system and has a strong impact on the $\mathrm{He}$ density distributions to be evaluated in the second step. To accurately describe the $\mathrm{He}-\mathrm{CNT}$ interaction in larger and longer carbon nanostructures, we introduce an additive pairwise atom-bond potential model. ${ }^{38}$ Its performance has been demonstrated recently for the interaction of $\mathrm{He}$ with graphene/graphite surfaces, ${ }^{38,39}$ and its applicability to nanotubes is illustrated in Figure 1 by direct comparison to the DFT-SAPT results for $\operatorname{CNT}(7,7)$. For brevity, a detailed analysis of the SAPT-based energy components has been shifted to the Supporting Information.

The additive pairwise potential model allows the generation of full three-dimensional potential energy surfaces (PESs) for the interaction of a $\mathrm{He}$ atom with a carbon nanotube of arbitrary size. We select nanotubes comprising 6 unit cells each, with helicity indices ranging from $(4,4)$ to $(9,9)$, giving six types with a length of $13.53 \AA$ and tube diameters in the range of 5.42-12.18 $\AA$. The results for one-dimensional scans along the tube axis and perpendicular to it are summarized in Figure 2. Zero energy is set to infinite distance between the $\mathrm{He}$ and the carbon nanotube. From this study it can be seen that the $(4,4)$ nanotube is too small for He propagation. The corresponding axial potential curve shows a bound state located at $8.82 \AA$, which lies approximately $2.06 \AA$ above the tube entrance. Its binding energy of $-160 \mathrm{~K}$ is just slightly lower than the adsorption energy anywhere outside on the tube surface $(-142 \mathrm{~K})$. The next larger nanotube $(5,5)$ is already large enough for helium penetration. Interestingly, this type shows 
Table 1. Characteristics of the Interaction between Helium and Carbon Nanotubes of Various Diameters ${ }^{a}$

\begin{tabular}{|c|c|c|c|c|c|c|c|}
\hline nanotube helicity & $(4,4)$ & $(5,5)$ & $(6,6)$ & $(7,7)$ & $(8,8)$ & $(9,9)$ & $(15,15)$ \\
\hline diameter $(\AA)$ & 5.42 & 6.74 & 8.14 & 9.47 & 10.85 & 12.18 & 20.33 \\
\hline$E_{\min }^{\mathrm{in}}(\mathrm{K})$ & - & -624 & -472 & -353 & -304 & -277 & -226 \\
\hline$E_{\min }^{\text {out }}(\mathrm{K})$ & -142 & -147 & -151 & -155 & -157 & -160 & -169 \\
\hline$r_{\min }^{\mathrm{in}}(\AA)$ & - & 0.00 & 0.56 & 1.44 & 2.15 & 2.86 & 6.96 \\
\hline$r_{\min }^{\text {out }}(\AA)$ & 5.86 & 6.53 & 7.23 & 7.90 & 8.58 & 9.27 & 13.33 \\
\hline$E_{\mathrm{zp}, r}^{\mathrm{in}}(\mathrm{K})$ & - & 81 & 18 & 44 & 48 & 49 & 48 \\
\hline$E_{\mathrm{zp}, z}^{\mathrm{in}}(\mathrm{K})$ & 31 & 3 & 3 & 2 & 2 & 2 & 1 \\
\hline$S_{(n, n)}(\mathrm{kcal} / \mathrm{mol})$ & -13.16 & -16.66 & -22.59 & -26.37 & -30.75 & -34.62 & -57.98 \\
\hline$f_{(n, n)}$ & - & 0.256 & 0.556 & 0.764 & 0.888 & 0.985 & 1.179 \\
\hline
\end{tabular}

${ }^{a}$ Approximate diameters and positions of the PES minima; adsorption energies at the PES minima; zero-point energies; solvation energies, $S_{(n, n)}$, for a complete submersion of the nanotubes in $\mathrm{He}_{2000}$; and the dimensionless filling factor, $f_{(n, n)}$.

the strongest $\mathrm{He}$ adsorption $(624 \mathrm{~K})$ of all nanotubes investigated, because its diameter allows for a symmetric attractive interaction with all neighboring carbon atoms on the inside. For the larger nanotubes, starting with the $(6,6)$ helicity, the PES minimum for adsorption on the inside moves away from the center and toward the inner surface. An equilibrium distance of 3.51 $\AA$ from the inner wall can be observed for the $(6,6)$ tube, which becomes slightly smaller for the larger tubes (3.23 $\AA$ for the largest in the series) as it slowly converges to the graphene adsorption value. The energies for external $\mathrm{He}$ adsorption are only weakly influenced by the nanotube size, growing from 142 to $160 \mathrm{~K}$ in the series.

From these cuts through the PES we can derive the zeropoint energy of a single $\mathrm{He}$ atom. Assuming a fully rigid nanotube, we have only the three degrees of freedom of the helium atom inside the channel. The zero-point energy for the vibrations along the radial and axial degrees of freedom can be easily determined by numerically solving the one-dimensional nuclear Schrödinger equation using the potential curves shown in Figure 2. These zero-point energies, obtained via a finitedifferences approach, are collected in Table 1. The strong confinement of the He atom upon adsorption inside the $(5,5)$ tube leads to a considerable zero-point energy in this geometry due to the steepness of the radial potential, which arises from the significant exchange-repulsion even at the nanotube center; see Supporting Information. After zero-point energy corrections are included, the lowest nuclear bound-states energies of the He atom for $(5,5)$ and $(6,6)$ nanotubes become comparable, but the He atom remains bound in both cases. Our results are in agreement with the experimental measurements of Ohba, ${ }^{20}$ showing that carbon nanotubes with diameters below $7 \AA$ do barely adsorb helium atoms at temperatures as low as 2 $\mathrm{K}$. Still, it is necessary to account for the $\mathrm{He}-\mathrm{He}$ interaction and the peculiarity of the superfluid quantum state in order to obtain better estimates of the helium filling factor. Following ref 20 , we define this factor as

$$
f_{(n, n)}=\rho_{\mathrm{av}} / \rho_{\text {bulk }}
$$

with $\rho_{\mathrm{av}}$ as the average helium density inside a $(n, n)$ nanotube and $\rho_{\text {bulk }}=0.02185 \AA^{-3}$ as the bulk value for liquid helium.

With the aim of obtaining a more realistic simulation of the adsorption process at temperatures below $2 \mathrm{~K}$, which is better described as the wetting of the carbon nanotubes in liquid, superfluid helium, the submersion process is characterized via orbital-free, bosonic helium density functional theory. Similar studies on the interaction of superfluid helium with various dopants have been performed by us in the past. ${ }^{40,41}$ Our selection of carbon nanotubes with diameters between 5 and 20
$\AA$ is placed inside a helium droplet consisting of $2000 \mathrm{He}$ atoms. The interactions between $\mathrm{He}$ and the carbon material are derived from the atom-bond potential model, providing full two-dimensional PESs in $z$ and $\rho$. A minimal corrugation appearing along the azimuthal degree of freedom is neglected in this study to preserve cylindric symmetry. However, we note that this corrugation can have noticeable effects on local phase transitions of the helium in situations of minimal coverage, as was demonstrated by Calvo in the case of the $\mathrm{C}_{60}^{+}-\mathrm{He}_{N}$ systems with $N<100$. $^{42}$

The free energy of a He nanodroplet with a carbon nanotube inside is calculated as follows. We apply orbital-free helium density functional theory (He-DFT) based on a slightly modified version of the Orsay-Trento density functional. ${ }^{16,29}$ Within this formulation, the helium nanodroplet is characterized as a condensate with all the ${ }^{4} \mathrm{He}$ atoms in the same $\mathrm{N}$ occupied state represented by an effective macroscopic wave function $\Psi(\mathbf{r})$ so that the total helium density is defined as $\rho(\mathbf{r})$ $=|\Psi(\mathbf{r})|^{2}$. We minimize the free energy, $F[\rho]$, a functional of this helium density $\rho$

$$
F[\rho]=E[\rho]+U_{\text {ext }}[\rho]-\mu N[\rho]-\mathbf{F} \cdot \mathbf{R}[\rho]
$$

with $E[\rho]$ denoting the Orsay-Trento density functional and $U_{\text {ext }}[\rho]$ representing the external potential introducing the interaction between droplet and nanotube

$$
U_{\text {ext }}[\rho]=\int \mathrm{d} \mathbf{r} \rho(\mathbf{r}) V_{\mathrm{ext}}^{\mathrm{He}-\mathrm{CNT}}(\mathbf{r})
$$

where $V_{\mathrm{ext}}^{\mathrm{He}-\mathrm{CNT}}$ is the atom-bond model potential for the $\mathrm{He}-$ CNT interaction. The two remaining terms of eq 2 reflect two constraints put on the minimization procedure: the conservation of $N$, the particle number, and $\mathbf{R}$, the He droplet mass center, appearing with their corresponding Lagrange parameters, the chemical potential $\mu$ and the retaining force $\mathbf{F}$, respectively. The density functional consists of a series of terms $^{29}$

$$
\begin{aligned}
E[\rho] & =\frac{\hbar^{2}}{2 m} \int \mathrm{d} \mathbf{r}(\nabla \sqrt{\rho(\mathbf{r})})^{2} \\
& +\frac{1}{2} \int \mathrm{d} \mathbf{r} \mathrm{d} \mathbf{r}^{\prime} \rho(\mathbf{r}) V_{\mathrm{LJ}}\left(\left|\mathbf{r}-\mathbf{r}^{\prime}\right|\right) \rho\left(\mathbf{r}^{\prime}\right) \\
& +\frac{c_{2}}{2} \int \mathrm{d} \mathbf{r} \rho(\mathbf{r}) \bar{\rho}(\mathbf{r})^{2}+\frac{c_{3}}{2} \int \mathrm{d} \mathbf{r} \rho(\mathbf{r}) \bar{\rho}(\mathbf{r})^{3} \\
& +C \int \mathrm{d} \mathbf{r}\left[1+\tanh \left(\beta\left\{\rho(\mathbf{r})-\rho_{m}\right\}\right)\right]
\end{aligned}
$$



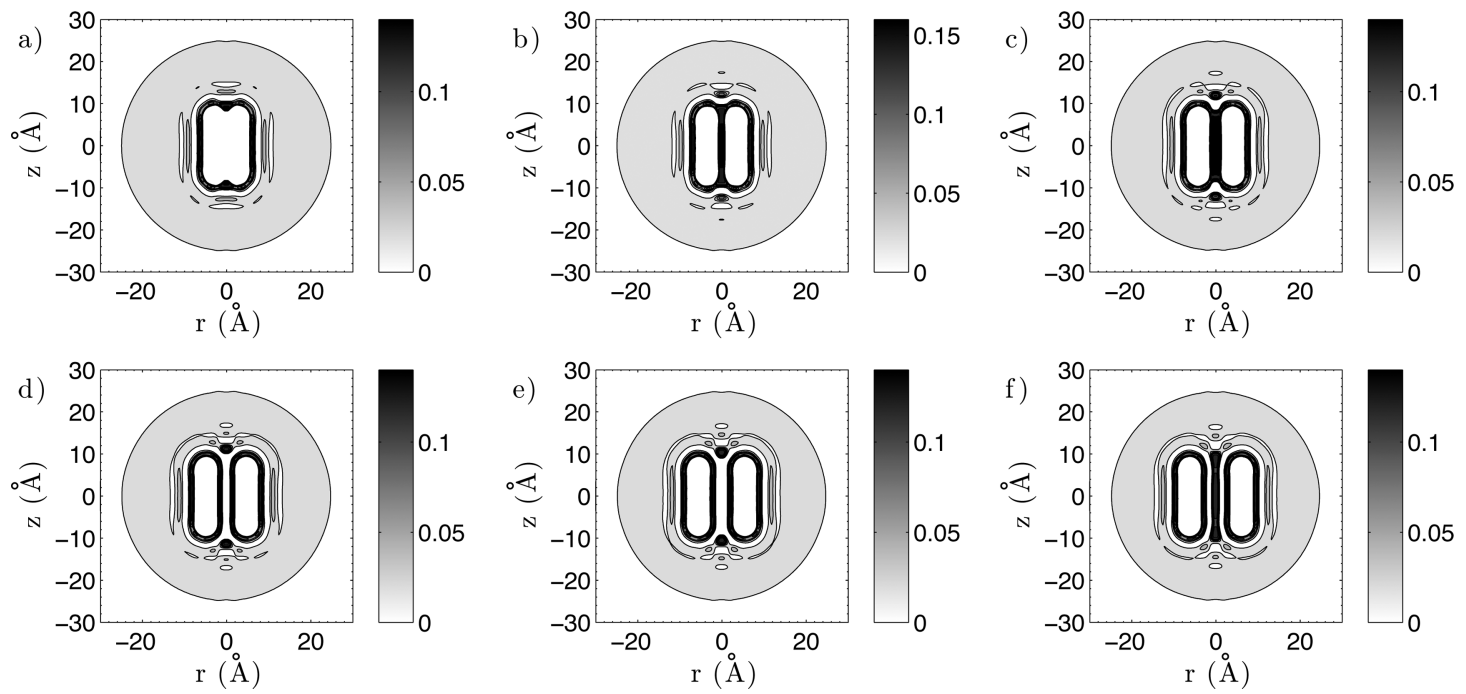

Figure 3. Contour plots of the helium density for fully submerged carbon nanotubes of various helicity $(n, n)$, from $n=4$ to 9 , labeled in ascending order from a to $\mathrm{f}$.
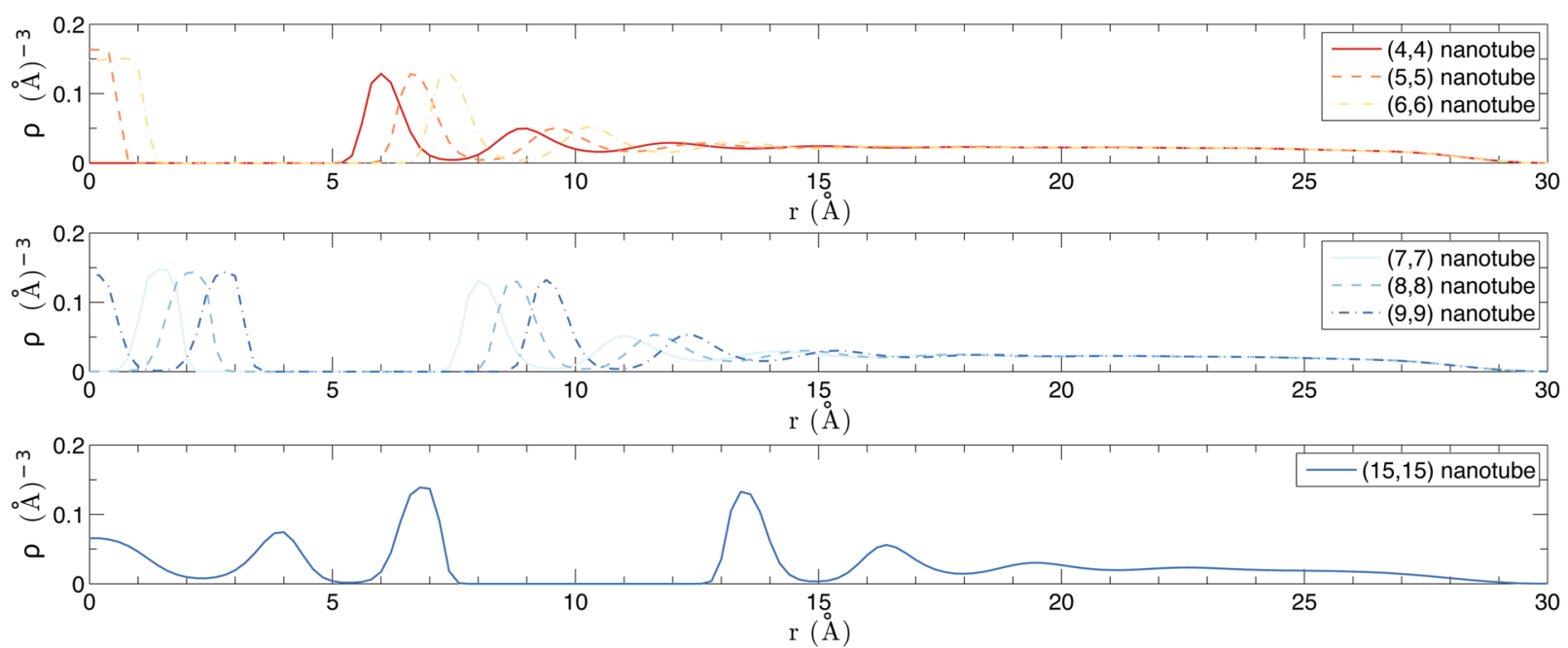

Figure 4. Radial distribution of the helium densities for fully immersed carbon nanotubes at $z=0$. The large areas of zero helium density represent the position of the carbon nanotube wall. Notice the strong oscillations of the density which are almost symmetric around the nanotube wall. These oscillations lead to regions of zero helium density even inside the larger tubes.

which can be identified as the quantum kinetic energy, the Lennard-Jones-type $\mathrm{He}-\mathrm{He}$ pair potential interaction energy, two short-range correlation energy contributions involving $\bar{\rho}$, a locally averaged density for a given sphere of radius $\bar{h}$, and a penalty term which forbids an extra pile-up of $\mathrm{He}$ density with $\rho_{m}$ as threshold value. For details, we refer to refs 29 and 30, in which this functional has been used to study the freezing transition of superfluid helium at high pressure. A timedependent version of it has been applied recently to underscore dispersion effects in the dynamics of helium nanodroplets on graphene. $^{43}$

As heliophilic dopants, the carbon nanotubes are supposed to sink into the helium completely. On the other side, depending on the tube radius, the flooding of the tube will come at the cost of increased total energy of the system. This interesting interplay of surface wetting and its avoidance due to increased zero-point energy for the confined helium is studied in detail. In Table 1 we provide solvation energies for all nanotubes, defined as the energy difference between full submersion and infinite distance between the helium droplet and the nanotube:

$$
S_{(n, n)}=E\left(\mathrm{He}_{2000}+\mathrm{C}_{(n, n)}\right)-E\left(\mathrm{He}_{2000}\right)
$$

All solvation energies in the table are negative, which confirms the assumption of a full submersion. However, a series of corresponding contour plots of the helium density for fully submerged carbon nanotubes (see Figure 3) reveals several interesting features: First, the relatively strong interaction between the helium and carbon structure leads to a first layer of very high helium density wrapped around the tube in a toroidal geometry in all cases except for the $(4,4)$ nanotube, where permeation is not possible. Second, two local maxima of $\mathrm{He}$ density appear along the axis near the tube entrance for all nanotubes except $(9,9)$. In the latter case, the diameter is large enough for yet another layer of high helium density to appear along the nanotube axis. This is better visible in the radial density plots provided in Figure 4, where it can be seen that the first few layers of highest helium density are separated by regions of complete zero density. Third, we find an almost constant peak-to-peak distance of approximately $2.8 \AA$, which is in excellent agreement with the experimentally measured 
thickness of helium layers on graphite (between 2.73 and 2.78 A). ${ }^{13}$ For demonstrative purposes we repeated our calculations with an even larger nanotube of helicity $(15,15)$ and the same tube length, because for this large diameter of $20.33 \AA$ yet another layer of solid helium appears in the center of the tube, giving a total of three layers inside the cylindrical confinement. The corresponding density contour plot is provided in Figure 5.

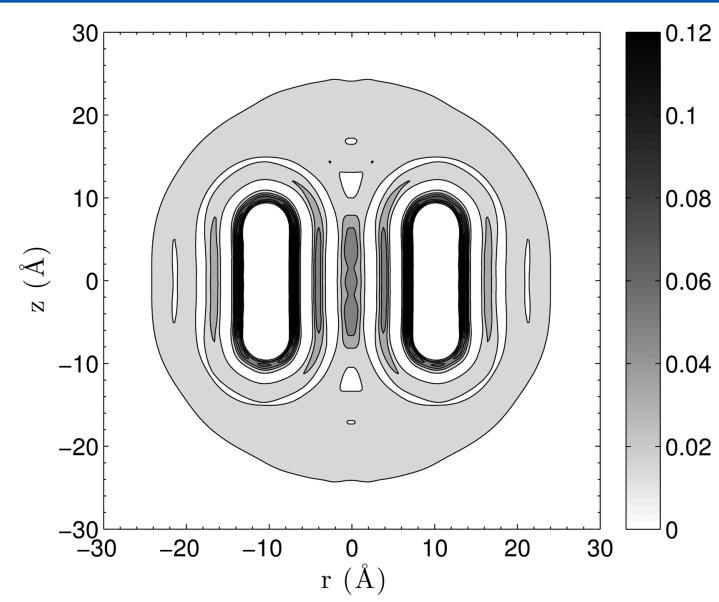

Figure 5. Contour plot of the helium density for the fully submerged carbon nanotube with helicity $(15,15)$. For this large tube, yet another layer of helium can pass through, leading to three radial shells in total.

Interestingly, the distance between the first (axial) and the second density peak is larger in this case (3.9 $\AA)$, which indicates a compromise between the tendency to form solid layers with optimal distance but also to fill up the nanotube volume.

The single peaks of the radial helium density in the $(5,5)$ and $(6,6)$ nanotubes have a width of approximately $1 \AA$, which corresponds to a single line of atoms. Therefore, these formats are candidates for the observation of a phase transition from the superfluid to a quasi-ordered, one-dimensional phase known as Luttinger liquid, ${ }^{44}$ which has been studied recently via quantum Monte Carlo simulations of helium flow through $\mathrm{Si}_{3} \mathrm{~N}_{4}$ nanopores. ${ }^{45}$ Interestingly, the results for radial densities in ref 45 show a similarly layered structure but differ from our findings in that there are no zones of zero density within the nanotubes. From this, one can assume that the surprising formation of helium cavities in carbon nanotubes is either a consequence of the different interaction potentials or related to the full embedding simulated in our studies versus the periodic approach of ref 45 . However, additional insights from the simplified atomic helium picture support the former assumption: By adding a single $\mathrm{He}$ atom to the center of the $(8,8)$ and $(9,9)$ tubes (i.e., adding the $\mathrm{He}-\mathrm{He}$ interaction to the radial PES shown in Figure 2), we obtain the modified PES for a second $\mathrm{He}$ atom. Zero-point energy calculations for a radial vibration of the second $\mathrm{He}$ atom in these modified PESs yield values of 130 and $65 \mathrm{~K}$ for the $(8,8)$ and the $(9,9)$ nanotube, respectively, while the corresponding PES minima are 220 and $290 \mathrm{~K}$. Notice that the inclusion of the second $\mathrm{He}$ atom at the center makes the effective $\mathrm{He}-$ nanotube interaction more attractive for the $(9,9)$ case while the opposite holds for the $(8,8)$ counterpart (see Table 1). Considering the double degeneracy of the radial vibration, there is no bound state possible in the $(8,8)$ nanotube, while there is in the larger $(9,9)$ nanotube.
To link our findings to experimental investigations based on adsorption measurements, we further provide the filling factors as defined in eq 1 . They are obtained by radial integration of the plotted helium densities and can be found in the last column of Table 1 . All values lie below 1 except for the largest nanotube $(15,15)$, which confirms the quenched adsorption of superfluid helium for nanotubes of $(9,9)$ helicity or smaller.

In summary, we applied bosonic, orbital-free He-DFT to investigate the change in total energy and in the helium density distribution of a superfluid helium droplet consisting of 2000 He atoms upon uptake of a single-walled carbon nanotube. The necessary $\mathrm{He}-\mathrm{CNT}$ interaction potential was derived via an additive pairwise model validated with DFT-SAPT calculations which allowed us to break down the intermolecular interaction into physically meaningful contributions. Depending on the diameter of the nanotube, the outer and inner walls of the nanotube are covered by one or more dense layers of helium upon full submersion. However, in contrast to quantum Monte Carlo studies on $\mathrm{Si}_{3} \mathrm{~N}_{4}$ nanotubes, ${ }^{45}$ the radial density oscillations show regions of complete zero density in our case. We attribute this unusual finding to the increased zeropoint energy for helium atoms inside the nanotube due to the confinement, paired with the tendency to keep the overall helium distribution minimally distorted. This is particularly evident for the $(8,8)$ nanotube, which shows an axial cavity even after full submersion into the $\mathrm{He}$ droplet. We further provide filling factors for a direct comparison to experimental studies based on helium flow or helium adsorption measurements. For carbon nanotubes with helicity $(9,9)$ or smaller, we predict a reduced uptake and quenched mobility of superfluid helium due to the layered structure and the cavities we observe in our simulations.

\section{ASSOCIATED CONTENT}

S Supporting Information

The Supporting Information is available free of charge on the ACS Publications website at DOI: 10.1021/acs.jpclett.6b02414.

Details of the potential model, results obtained with the DFT-SAPT method, and a vibrational analysis of $\mathrm{He}$ atoms confined in carbon nanotubes (PDF)

\section{AUTHOR INFORMATION}

\section{Corresponding Authors}

*E-mail: andreas.w.hauser@gmail.com.

*E-mail: Pilar.deLara.Castells@csic.es.

ORCID ${ }^{\circledR}$

Andreas W. Hauser: 0000-0001-6918-3106

María Pilar de Lara-Castells: 0000-0001-8697-5770

Notes

The authors declare no competing financial interest.

\section{ACKNOWLEDGMENTS}

This work has been supported by the COST Action CM1405 "Molecules in Motion (MOLIM)". M.P.d.L.-C. gratefully acknowledges support from MINECO (Spain) under Grant MAT2016-75354-P and thanks the CTI (CSIC) and CESGA supercomputer facilities (Spain) for the resources provided.

\section{REFERENCES}

(1) Baughman, R. H.; Zakhidov, A. A.; de Heer, W. A. Carbon Nanotubes-the Route Toward Applications. Science 2002, 297, 787792. 
(2) Iijima, S.; Ichihashi, T. Single-shell Carbon Nanotubes of 1-nm Diameter. Nature 1993, 363, 603-605.

(3) R, S.; Dresselhaus, G.; Dresselhaus, M. Physical Properties of Carbon Nanotubes; Imperial College Press: London, 1998; p 259.

(4) Kowalczyk, P.; Terzyk, A. P.; Gauden, P. A.; Furmaniak, S.; Kaneko, K.; Miller, T. F., III Nuclear Quantum Effects in the Layering and Diffusion of Hydrogen Isotopes in Carbon Nanotubes. J. Phys. Chem. Lett. 2015, 6, 3367-3372.

(5) Schrier, J. Helium Separation Using Porous Graphene Membranes. J. Phys. Chem. Lett. 2010, 1, 2284-2287.

(6) Hauser, A. W.; Schwerdtfeger, P. Nanoporous Graphene Membranes for Efficient ${ }^{3} \mathrm{He} /{ }^{4} \mathrm{He}$ Separation. J. Phys. Chem. Lett. 2012, 3, 209-213.

(7) Hauser, A. W.; Schrier, J.; Schwerdtfeger, P. Helium Tunneling through Nitrogen-Functionalized Graphene Pores: Pressure- and Temperature-Driven Approaches to Isotope Separation. J. Phys. Chem. C 2012, 116, 10819-10827.

(8) Bartolomei, M.; Carmona-Novillo, E.; Hernández, M. I.; Campos-Martínez, J.; Pirani, F.; Giorgi, G. Graphdiyne Pores: "Ad Hoc" Openings for Helium Separation Applications. J. Phys. Chem. C 2014, 118, 29966-29972.

(9) Hernández, M. I.; Bartolomei, M.; Campos-Martínez, J. Transmission of Helium Isotopes through Graphdiyne Pores: Tunneling versus Zero Point Energy Effects. J. Phys. Chem. A 2015, 119, 10743-10749.

(10) Niimura, S.; Fujimori, T.; Minami, D.; Hattori, Y.; Abrams, L.; Corbin, D.; Hata, K.; Kaneko, K. Dynamic Quantum Molecular Sieving Separation of $\mathrm{D}_{2}$ from $\mathrm{H}_{2} \mathrm{D}_{2}$ Mixture with Nanoporous Materials. J. Am. Chem. Soc. 2012, 134, 18483-18486. PMID: 23116187.

(11) Craig, I. R; Manolopoulos, D. E. Quantum Statistics and Classical Mechanics: Real Time Correlation Functions from Ring Polymer Molecular Dynamics. J. Chem. Phys. 2004, 121, 3368.

(12) Habershon, S.; Manolopoulos, D. E.; Markland, T. E.; Miller, T. F., III Ring-Polymer Molecular Dynamics: Quantum Effects in Chemical Dynamics from Classical Trajectories in an Extended Phase Space. Annu. Rev. Phys. Chem. 2013, 64, 387-413. PMID: 23298242

(13) Carneiro, K.; Passell, L.; Thomlinson, W.; Taub, H. NeutronDiffraction Study of the Solid Layers at the Liquid-Solid Boundary in ${ }^{4} \mathrm{He}$ Films Adsorbed on Graphite. Phys. Rev. B: Condens. Matter Mater. Phys. 1981, 24, 1170-1176.

(14) Cheng, E.; Cole, M. W.; Saam, W. F.; Treiner, J. Phase Transitions in Multilayer Helium Films. Phys. Rev. B: Condens. Matter Mater. Phys. 1992, 46, 13967-13982.

(15) Clements, B. E.; Krotscheck, E.; Lauter, H. J. Growth Instability in Helium Films. Phys. Rev. Lett. 1993, 70, 1287-1290.

(16) Dalfovo, F.; Lastri, A.; Pricaupenko, L.; Stringari, S.; Treiner, J. Structural and Dynamical Properties of Superfluid Helium: A DensityFunctional Approach. Phys. Rev. B: Condens. Matter Mater. Phys. 1995, 52, 1193.

(17) Krotscheck, E.; Apaja, V.; Rimnac, A.; Zillich, R. Quantum Liquids in Confinement: the Microscopic View. J. Phys.: Condens. Matter 2003, 15, S95.

(18) Pierce, M.; Manousakis, E. Quantum Films Adsorbed on Graphite: Third and Fourth Helium Layers. Phys. Rev. B: Condens. Matter Mater. Phys. 2001, 63, 144524.

(19) Savard, M.; Dauphinais, G.; Gervais, G. Hydrodynamics of Superfluid Helium in a Single Nanohole. Phys. Rev. Lett. 2011, 107, 254501.

(20) Ohba, T. Limited Quantum Helium Transportation through Nano-channels by Quantum Fluctuation. Sci. Rep. 2016, 6, 28992.

(21) Clements, B. E.; Epstein, J. L.; Krotscheck, E.; Saarela, M. Structure of Boson Quantum Films. Phys. Rev. B: Condens. Matter Mater. Phys. 1993, 48, 7450-7470.

(22) Saslow, W. M.; Agnolet, G.; Campbell, C. E.; Clements, B. E.; Krotscheck, E. Theory of First-Order Layering Transitions in Thin Helium Films. Phys. Rev. B: Condens. Matter Mater. Phys. 1996, 54, 6532-6538.
(23) Campbell, C. E.; Clements, B. E.; Krotscheck, E.; Saarela, M. Thermodynamics of Boson quantum films. Phys. Rev. B: Condens. Matter Mater. Phys. 1997, 55, 3769-3791.

(24) Gottlieb, J. M.; Bruch, L. W. Ground State of Monolayer ${ }^{4} \mathrm{He} /$ Graphite: Quantum Liquid versus Quantum Solid. Phys. Rev. B: Condens. Matter Mater. Phys. 1993, 48, 3943-3948.

(25) Wagner, M.; Ceperley, D. M. Path Integral Monte Carlo Simulations of Thin ${ }^{4} \mathrm{He}$ Films on a $\mathrm{H}_{2}$ Surface. J. Low Temp. Phys. 1994, 94, 185-217.

(26) Pierce, M.; Manousakis, E. Phase Diagram of Second Layer of ${ }^{4}$ He Adsorbed on Graphite. Phys. Rev. Lett. 1998, 81, 156-159.

(27) Ceperley, D. M.; Manousakis, E. Path Integral Monte Carlo Applications to Quantum Fluids in Confined Geometries. J. Chem. Phys. 2001, 115, 10111.

(28) Cheng, E.; Cole, M. W.; Dupont-Roc, J.; Saam, W. F.; Treiner, J. Novel Wetting Behavior in Quantum Films. Rev. Mod. Phys. 1993, 65, 557-567.

(29) Ancilotto, F.; Barranco, M.; Caupin, F.; Mayol, R.; Pi, M. Freezing of ${ }^{4} \mathrm{He}$ and its Liquid-Solid Interface from Density Functional Theory. Phys. Rev. B: Condens. Matter Mater. Phys. 2005, 72, 214522.

(30) Caupin, F.; Ancilotto, F.; Barranco, M.; Mayol, R.; Pi, M. Freezing of Helium-4: Comparison of Different Density Functional Approaches. J. Low Temp. Phys. 2007, 148, 731-736.

(31) Toennies, J. P.; Vilesov, A. F. Superfluid Helium Droplets: a Uniquely Cold Nanomatrix for Molecules and Molecular Complexes. Angew. Chem., Int. Ed. 2004, 43, 2622-2648.

(32) Tiggesbäumker, J.; Stienkemeier, F. Formation and Properties of Metal Clusters Isolated in Helium Droplets. Phys. Chem. Chem. Phys. 2007, 9, 4748-4770.

(33) Callegari, C.; Ernst, W. E. In Handbook of High Resolution Spectroscopy; Merkt, F., Quack, M., Eds.; John Wiley \& Sons: Chichester, U.K., 2011.

(34) Mudrich, M.; Stienkemeier, F. Photoionisaton of Pure and Doped Helium Nanodroplets. Int. Rev. Phys. Chem. 2014, 33, 301339.

(35) Misquitta, A. J.; Jeziorski, B.; Szalewicz, K. Dispersion Energy from Density-Functionl Theory Description of Monomers. Phys. Rev. Lett. 2003, 91, 033201.

(36) Heßelmann, A.; Jansen, G. Intermolecular Dispersion Energies from Time-Dependent Density Functional Theory. Chem. Phys. Lett. 2003, 367, 778-784.

(37) Werner, H. J.; Knowles, P. J.; Knizia, G.; Manby, F. R.; Schütz, M.; Celani, P.; Korona, T.; Lindh, R.; Mitrushchenkov, A. O.; Rauhut, G. et al. MOLPRO, version 2012.1, a package of ab initio programs; http://www.molpro.net.

(38) Bartolomei, M.; Carmona-Novillo, E.; Hernández, M. I.; Campos-Martínez, J.; Pirani, F. Global Potentials for the Interaction between Rare Gases and Graphene-Based Surfaces: An Atom-Bond Pairwise Additive Representation. J. Phys. Chem. C 2013, 117, 1051210522.

(39) de Lara-Castells, M. P.; Bartolomei, M.; Mitrushchenkov, A. O.; Stoll, H. Transferability and Accuracy by Combining Dispersionless Density Functional and Incremental Post-Hartree-Fock Theories: Noble gases Adsorption on Coronene/Graphene/Graphite Surfaces. J. Chem. Phys. 2015, 143, 194701.

(40) Hauser, A. W.; de Lara-Castells, M. P. Spatial Quenching of a Molecular Charge-Transfer Process in a Quantum Fluid: The $\mathrm{Cs}_{x}-\mathrm{C}_{60}$ Reaction in Superfluid Helium Nanodroplets. Phys. Chem. Chem. Phys., submitted for publication, 2016.

(41) Poms, J.; Hauser, A. W.; Ernst, W. E. Helium Nanodroplets Doped with Xenon and Rubidium Atoms: a Case Study of van der Waals Interactions Between Heliophilic and Heliophobic Dopants. Phys. Chem. Chem. Phys. 2012, 14, 15158-15165.

(42) Calvo, F. Size-Induced Melting and Reentrant Freezing in Fullerene-Doped Helium Clusters. Phys. Rev. B: Condens. Matter Mater. Phys. 2012, 85, 060502.

(43) de Lara-Castells, M. P.; Stoll, H.; Civalleri, B.; Causà, M.; Voloshina, E.; Mitrushchenkov, A. O.; Pi, M. Communication: A Combined Periodic Density Functional and Incremental Wave- 
Function-based Approach for the Dispersion-Accounting TimeResolved Dynamics of ${ }^{4} \mathrm{He}$ Nanodroplets on Surfaces: ${ }^{4} \mathrm{He} /$ Graphene. J. Chem. Phys. 2014, 141, 151102.

(44) Haldane, F. D. M. Effective Harmonic-Fluid Approach to LowEnergy Properties of One-Dimensional Quantum Fluids. Phys. Rev. Lett. 1981, 47, 1840-1843.

(45) Del Maestro, A.; Boninsegni, M.; Affleck, I. ${ }^{4} \mathrm{He}$ Luttinger Liquid in Nanopores. Phys. Rev. Lett. 2011, 106, 105303. 